\title{
Acknowledgement to Reviewers of MCA in 2017
}

\author{
MCA Editorial Office \\ MDPI AG, St. Alban-Anlage 66, 4052 Basel, Switzerland \\ Published: 16 January 2018
}

Peer review is an essential part in the publication process, ensuring that MCA (Mathematical and Computational Applications) maintains high quality standards for its published papers. In 2017, a total of 43 papers were published in the journal. Thanks to the cooperation of our reviewers, the median time to first decision was 46 days and the median time to publication was 77 days. The editors would like to express their sincere gratitude to the following reviewers for their time and dedication in 2017:

\begin{tabular}{|c|c|}
\hline Abbasbandy, Saeid & Chen, Wen \\
\hline Abel, Mati & Chen, Wenli \\
\hline Akram, Muhammad & Chen, Wenyu \\
\hline Ali, Montaz & Choobineh, F. \\
\hline Amat, Sergio & Clayton, John D. \\
\hline Antunes, Nelson & Climente-Alarcon, Vicente \\
\hline Ata, Seisuke & Conti, Costanza \\
\hline Außenhofer, Lydia & Costantino, Nicola \\
\hline Avdonin, Sergei & Cruz, Roberto \\
\hline Badoual, Anaïs & Csendes, Tibor \\
\hline Bagirov, Adil & Dabo-Niang, Sophie \\
\hline Bai, Chuanzhi & Dai, Chao-Qing \\
\hline Baker, Thar & Dandil, Emre \\
\hline Bambozzi, Federico & D'Andreagiovanni, Fabio \\
\hline Bao, $\mathrm{Yi}$ & Davison, Matt \\
\hline Barton, Michael & De Carne, Giovanni \\
\hline Bartz-Beielstein, Thomas & De Fenza, Angelo \\
\hline Baskonus, Haci Mehmet & De Macedo Mourelle, Luiza \\
\hline Birken, Philipp & Dei, Michele \\
\hline Branch, John William & Delefortrie, Guillaume \\
\hline Breitkopf, Cornelia & Delgado, Jorge \\
\hline Bruneel, Herwig & Dell'Accio, Francesco \\
\hline Bui, Tinh Quoc & Demidova, Liliya \\
\hline Byrd, Richard & Demirhan, Ayşe \\
\hline Calder, Jeff & Dimitrov, Darko \\
\hline Calderón Moreno, Maria Del Carmen & Dong, Guangneng \\
\hline Campi, Cristina & Ehrhardt, Matthias \\
\hline Campoamor Stursberg, Rutwig & Emmert, Steffen \\
\hline Can, Mehmet & Enright, Wayne \\
\hline Carletti, Vincenzo & Faez, Karim \\
\hline Carrasco, Paulo & Fan, Chia-Ming \\
\hline Chen, Alex & Fantuzzi, Nicholas \\
\hline Chen, Bo & Ferreira da Silva, Paulo \\
\hline Chen, Chun-I & Fra,ckiewicz, Piotr \\
\hline Chen, Tinggui & Gaeta, Giuseppe \\
\hline
\end{tabular}


García Gonzalo, Maria Esperanza

Ghaedi, A.M.

Gibali, Aviv

Glowacz, Adam

Goffard, Pierre-Olivier

Gökdoğan, Ahmet

Goldman, Ron

Gopalan, Anandha

Gucma, Lucjan

Gulasekaran, Rajaguru

Gunawan, Indra

Hadjidimos, Apostolos

Hamza, Ben

Heidergott, Bernd

Hong, Shihuang

Hormann, Kai

Hsiao, Kai-Long

Huber, Michael

Huyer, Waltraud

Jacobs, Byron

Jain, Prashant K.

Janssens, Gerrit K.

Jasperneite, Jürgen

Jator, Samuel N.

Kanduč, Tadej

Katona, Gabriel

$\mathrm{Ke}$, Changhong

Kennedy, David

Khan, Akhtar A.

Koch, Othmar

Koyuncu, Fatih

Kulkarni, Anand J.

Lachman-Senesh, Noa

Lau, Denvid

Lee, Seongkyu

Lee, Tsung-Hsueh

Leung, Carson

Li, Leida

Lian, Yanping

Linders, Daniël

Liu, Shancun

Liu, Sifeng

Lopez-Iturri, Peio

Löpker, Andreas

Luan, Vu Thai

Luchko, Yuri

$\mathrm{Ma}$, Changfeng

$\mathrm{Ma}$, Hongyan

Ma, Wenxiu

Majstorović, Snježana

Mao, Qibo

Mazucheli, Josmar
Melenka, Garrett

Melo, Jefferson

Mendes, Jérôme

Meng, Zhaoliang

Miletic, Maja

Mondal, Sankar

Muzzioli, Silvia

Nadri, Madiha

Nævdal, Eric

Naseer, Noman

Navarro Peris, Emilio

Nazir, Sajjad

Neytcheva, Maya G.

Nguyen, Linh

Nie, Shouping

Nieto, Juan J.

Nikas, George K.

Paquette, Julie

Parreiras, Roberta Oliveira

Pascoal, Marta

Paternoster, Beatrice

Pau, Giovanni

Paulides, Johannes

Pedersen, Jesper Lund

Pellegrino, Roberta

Peterson, Allan C.

Philipp, Mucha

Pickup, David

Piot-Lepetit, Isabelle

Pisano, Aurora Angela

Price, Christopher John

Puche-Panadero, Rubén

Qiu, Jingmei

Rahnejat, Homer

Raman, Gireesh

Ramos, Célia M. Q.

Ramos, Higinio

Rashidi, Mohammd Mehdi

Rivera, Jaime

Rosaci, Domenico

Sadowski, Lukasz

Samanta, GuruPrasad

Samodivkin, Vladimir

Santini, Francesco

Schütze, Oliver

Sessa, Valentina

Shaat, Mohamed Ibrahim

Shen, Shoufeng

Shengjun, Liu

Shifrin, Theodore

Silva, Hugo

Simsek, Yilmaz 
Sladkowski, Jan

Solachidis, Vassilios

Solano, Juan Pedro

Sostak, Alexander

Srivastava, Hari M

Stanovský, Petr

Strnad, Damjan

Subramanian, Ramanathan

Sukhorukova, Nadezda

Svob, Andrea

Tahmasebi, Pejman

Tatar, Nasser-Eddine

Theodoulidis, Theodoros

Tiakas, Eleftherios

Towers, Isaac

Transtrum, Mark K.

Tsai, Yuan-Yu

Tsitouras, Charalampos

Tzoumas, Michael G.

Uslar, Mathias

Van Casteren, Dave

Van Do, Tien

Vantorre, Marc

Venable, Kristen Brent

Volkovich, Zeew

Wang, Binbin

Wang, Min

Wang, Wansheng

Wang, Xueju

Wassermann, Alfred

Weber, Gerhard-Wilhelm
Wen, Kunli

Wibowo, Andreas

Woźniak, Marcin

Wu, Cheng-Wei

$\mathrm{Wu}$, Ming Lu

Wu, Qingbiao

$\mathrm{Wu}$, Yuanming

$\mathrm{Xu}$, Gang

$\mathrm{Xu}$, Hang

Yacout, Soumaya

Yang, Fan

Yang, Tian-Zhi

Yang, Xin-She

$\mathrm{Yu}$, Chun-Xiao

Yu, Feng

Yu, Wenwu

Zeng, Wenhan

Zeng, Zhiguo

Zhang, Chao

Zhang, Jiwei

Zhang, Liping

Zhang, Shuo

Zhang, Weiguang

Zhao, Jingjun

Zhao, Xianbo

Zhao, Ya-Pu

Zhou, Bo

Zhou, Huachun

Zhou, Kai

Zuo, Wenjie

(C) 2018 by the authors. Licensee MDPI, Basel, Switzerland. This article is an open access article distributed under the terms and conditions of the Creative Commons Attribution (CC BY) license (http://creativecommons.org/licenses/by/4.0/). 